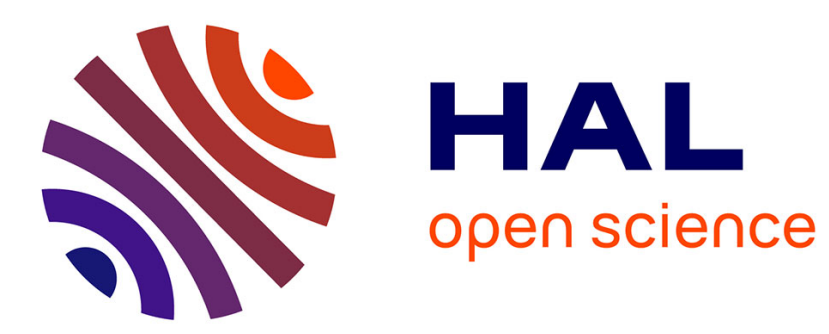

\title{
Extracellular versus Intracellular Degradation of Nanostructured Silica Particles
}

\author{
Yupeng Shi, Christophe Hélary, Bernard Haye, T. Coradin
}

\section{To cite this version:}

Yupeng Shi, Christophe Hélary, Bernard Haye, T. Coradin. Extracellular versus Intracellular Degradation of Nanostructured Silica Particles. Langmuir, 2018, 34 (1), pp.406-415. 10.1021/acs.langmuir.7b03980 . hal-01668691

\section{HAL Id: hal-01668691 \\ https://hal.sorbonne-universite.fr/hal-01668691}

Submitted on 20 Dec 2017

HAL is a multi-disciplinary open access archive for the deposit and dissemination of scientific research documents, whether they are published or not. The documents may come from teaching and research institutions in France or abroad, or from public or private research centers.
L'archive ouverte pluridisciplinaire HAL, est destinée au dépôt et à la diffusion de documents scientifiques de niveau recherche, publiés ou non, émanant des établissements d'enseignement et de recherche français ou étrangers, des laboratoires publics ou privés. 


\section{Article}

Subscriber access provided by BUPMC - Bibliothèque Universitaire Pierre et Marie Curie

\section{Extracellular vs. Intracellular Degradation of Nanostructured Silica Particles}

Yupeng Shi, Christophe Helary, Bernard Haye, and Thibaud Coradin

Langmuir, Just Accepted Manuscript • DOI: 10.1021/acs.langmuir.7b03980 • Publication Date (Web): 11 Dec 2017

Downloaded from http://pubs.acs.org on December 12, 2017

\section{Just Accepted}

"Just Accepted" manuscripts have been peer-reviewed and accepted for publication. They are posted online prior to technical editing, formatting for publication and author proofing. The American Chemical Society provides "Just Accepted" as a free service to the research community to expedite the dissemination of scientific material as soon as possible after acceptance. "Just Accepted" manuscripts appear in full in PDF format accompanied by an HTML abstract. "Just Accepted" manuscripts have been fully peer reviewed, but should not be considered the official version of record. They are accessible to all readers and citable by the Digital Object Identifier (DOIß). "Just Accepted" is an optional service offered to authors. Therefore, the "Just Accepted" Web site may not include all articles that will be published in the journal. After a manuscript is technically edited and formatted, it will be removed from the "Just Accepted" Web site and published as an ASAP article. Note that technical editing may introduce minor changes to the manuscript text and/or graphics which could affect content, and all legal disclaimers and ethical guidelines that apply to the journal pertain. ACS cannot be held responsible for errors or consequences arising from the use of information contained in these "Just Accepted" manuscripts. 


\title{
Extracellular vs. Intracellular Degradation of Nanostructured Silica Particles
}

\author{
Yupeng Shi, Christophe Hélary, Bernard Haye and Thibaud Coradin* \\ Sorbonne Universités, UPMC Univ Paris 06, CNRS, UMR 7574, Laboratoire de Chimie de \\ la Matière Condensée de Paris, F-75005 Paris, France
}

\begin{abstract}
Silica nanoparticles appear as promising drug carriers for intracellular delivery. However the mechanisms by which they are degraded within cells remain largely unknown. In this context, we have prepared three types of PEGylated fluorescent silica nanoparticles with various internal structures (core-shell bio-composite, multilayered and hollow mesoporous) and studied their degradation in buffer, in culture medium and in contact with human dermal fibroblasts. All particles were prone to dissolve in solution, leading to an increase of porosity and/or precipitation of new colloids and eventually fragmentation, with a faster rate in medium compared to buffer. All particles were also uptaken by cells without significant cytotoxic effect. Their intracellular degradation occurred faster than in suspension but following almost similar dissolution mechanisms. These results strongly suggest that, in these conditions, silica nanoparticles must be primarily considered as hydrolytically degraded and not biodegraded, a point of importance for their future application in drug delivery.
\end{abstract}




\section{INTRODUCTION}

The unique features of nanomaterials have led to their rapid development in the biomedical field, for example in the delivery of drugs and genes, or for bio-imaging and diagnosis. ${ }^{1-4}$ In this context, understanding the interactions between nanoparticles and cells is crucial to elucidate the true nature of the nanoparticle-mediated biological effects.

In particular, although efforts have been devoted to discover the factors that affect the cellular uptake, toxicity and degradation of nanomaterials, ${ }^{5-8}$ a detailed understanding of their intracellular fate remains a true challenge.

Silica nanomaterials have been used on an industrial scale for a long time in various technological fields. ${ }^{9}$ They also have a variety of unique properties, such as versatile synthesis, controllable size and architecture, easy surface modification, and relatively stable and homogeneous chemical composition, which endow them with many advantages for biomedical applications. ${ }^{10}$ A number of silica-based nanomaterials with engineered shape, structure and surface modification, such as solid nanoparticles/rod, mesoporous, hollow, multilayered, core-shell nanostructure as well as bio-composite nanomaterials, have been synthesized and evaluated as drug/gene delivery systems. ${ }^{11-16}$ However, in order to use them for intracellular delivery, successive steps of internalization, cellular trafficking, drug release and externalization should be finely controlled. ${ }^{17-19}$ Among these, the control of intracellular behavior of the nanoparticles remains highly challenging, especially because the question whether mammalian cells 
exhibit specific biological mechanisms to degrade silica remains open. ${ }^{20,21}$ Indeed, the degradation of silica nanoparticles in biologically-relevant conditions has been studied in details and recently reviewed by Croissant et al. ${ }^{21}$ The accumulated evidences point out the dynamic equilibrium between silica dissolution and re-deposition, which is influenced by factors such as $\mathrm{pH}$, temperature, silica concentration and porosity. ${ }^{22-25}$ Dove et al. demonstrated that the primary dissolution event consists in the detachment of a $\left[\mathrm{SiO}_{4}\right]$ tetrahedron from the silica surface, ${ }^{26}$ that is expected to be influenced by (i) the coordination number of $\mathrm{Si}$ atoms at the surface, (ii) the stability and reactivity of the detached species and (iii) the extent of the surface and its accessibility to water. ${ }^{27}$ As reviewed by Ehrlich et al., ${ }^{28}$ the first two parameters are related to the condensation degree of the silica network and the relative abundance of protonated, neutral and deprotonated silanols while the last parameter is controlled by the specific surface area of the system and the presence of adsorbed molecules. For instance, it has been demonstrated that the degradation rate of silica gels with similar specific surface area could be tuned by their condensation degree, ${ }^{29}$ and that the presence of a protein coating could slow down the dissolution process. ${ }^{30}$ In parallel, Kuroda et al. showed that mesoporous particles dissolve faster than plain silica particles of the same sizes. ${ }^{31}$ The enhanced dissolution rate of silica particles with increasing $\mathrm{pH}$ and/or in the presence of phosphate ions has also been reported at several occasions. ${ }^{21,27,28,32}$ Concerning the possible redeposition of the dissolved species, a comparative study of two types of mesoporous silica nanoparticles showed that the degradation of MCM-41-type 
mesoporous silica occurred from the outer surface, while for SBA-15 nanoparticles a decrease in microporosity and an increase in mesoporosity suggested a complex inner solubilization/recondensation process. $^{33}$ Other studies have suggested that the degradation of mesoporous nanoparticles may simultaneously proceed from the outer and the inner surfaces. ${ }^{34,35}$

Much less is known about the intracellular fate of silica nanoparticles. Earlier reports on the evolution of bionanocomposite silica-biopolymer nanoparticles within the intracellular space of mammalian cells have suggested that, after $24 \mathrm{~h}$, only the bio-organic fraction was degraded while the inorganic part remained intact. ${ }^{36,37}$ Further experiments performed with mesoporous nanoparticles evidenced that their cellular uptake was followed by their exocytosis. In addition the released particles were associated with proteins located in the cellular membrane or involved in the vesicular trafficking. ${ }^{38}$ However no modification in particle size or morphology was reported. A first insight in the intracellular dissolution pathway of plain silica nanoparticles was obtained by Quignard et al. through a combination of TEM imaging and identification of the products of their degradation. ${ }^{39}$ A decrease of particle size was observed and attributed to surface erosion as a result of the dissolution equilibrium of silica. Since then, further evidences of the ability of silica to dissolve intracellularly were accumulated. ${ }^{40-43}$ Yet, considering the complexity of the accompanying trafficking events, that can include $\mathrm{pH}$ modifications and protein deposition, defining the exact conditions in which silica particles are degraded remains highly challenging. 
At present, there are mainly three strategies to load small molecular drugs and biological macromolecules within silica nanomaterials. The first is to mix the drugs with the silica precursor to form nanocomposites. ${ }^{44,45}$ The second is through the layer-by-layer method. ${ }^{46,47}$ The third involves the impregnation of mesoporous or hollow silica nanoparticles. $^{48,49}$ As far as we know, there is no report comparing the degradation behavior of these three types of silica nanoparticles in biologically-relevant conditions. With this purpose, we have prepared PEGylated fluorescent silica particles with different internal structures (bio-composite, multilayered and hollow mesoporous) and studied their time evolution in abiotic (phosphate buffer, culture medium) conditions and then in the presence of normal human dermal fibroblasts. Structural degradation and dissolution kinetics of the nanoparticles were studied in parallel with their cytotoxicity. The here-gathered data suggest that, for these systems, the intracellular fate of silica nanoparticles can be explained on the sole basis of physico-chemical processes. This provides new and strong evidences that, in the intracellular space, silica nanoparticles should be considered as hydrolytically-degradable rather than biodegradable materials, a result of great consequences for the design of silica-based biomaterials.

\section{MATERIALS AND METHODS}

Chemicals. Tetraethyl orthosilicate (TEOS), (3-Aminopropyl)triethoxysilane (APTES), Branched polyethyleneimine (PEI, $25 \mathrm{kDa}$ ), Bovine serum albumin (BSA, $66 \mathrm{kDa}$ ), Cetyl trimethylammonium bromide (CTAB) were obtained from 
Sigma-Aldrich. Fluorescein Isothiocyante (Isomer I) (FITC), N-hydroxysuccinimide (NHS) and 1-ethyl-3-(3-dimethylaminopropyl)-carbodiimide (EDC) were obtained from Alfa Aesar. Methoxypolyethylene glycol acetic acid N-succinimidyl ester (NHS-PEG $5000-M A L)$ dimethylsulfoxide (DMSO), triethanolamine (TEA) and ethylenediamine tetraacetic acid (EDTA) were obtained from Sigma-Aldrich. Dubelcco's Modified Eagle Medium (DMEM) and Fetal Calf Serum (FCS) was purchased from Gibco BRL. Unless specified, all the commercial products were used without further purification.

\section{$\begin{array}{llllll}\text { Synthesis of } & \text { PEGylated } & \mathrm{BSA} @ \mathrm{SiO}_{2} & \text { Nanoparticles } & \text { (BSNPs). } & \mathrm{BSA} @ \mathrm{SiO}_{2}\end{array}$} nanoparticles were synthesized via a modified Stöber method. ${ }^{50}$ First, $30 \mathrm{mg}$ BSA were diluted in $3 \mathrm{~mL}$ deionized (DI) water and then added to $20 \mathrm{~mL}$ of ethanol containing 2 $\mathrm{mL}$ ammonia. After 5 minutes, $1 \mathrm{~mL}$ TEOS was added under stirring for 1 hour. Lastly, $50 \mu \mathrm{L}$ APTES-FITC was added three times every two hours, followed by vigorous stirring for 24 hours. Another $50 \mu \mathrm{L}$ APTES was added and after for 24 hours under stirring, amine-functionalized $\mathrm{BSA} @ \mathrm{SiO}_{2}-\mathrm{NH}_{2}$ particles were obtained, recovered by centrifugation and washed with ethanol twice. Next, BSA@ $\mathrm{SiO}_{2}-\mathrm{NH}_{2}$ were re-dispersed in $5 \mathrm{~mL}$ of PBS (phosphate buffer saline, $1 \mathrm{X}$ ), and $8 \mathrm{mg}$ of $\mathrm{NHS}_{-\mathrm{PEG}}{ }_{5000}-\mathrm{MAL}$ was added. $^{51}$ The mixture was then stirred at room temperature for 2 hours before harvesting PEGylated particles by centrifugation. 


\section{Synthesis of PEGylated Hollow Mesoporous Silica Nanoparticles (HSNPs).}

Fluorescent uniform $\sim 100 \mathrm{~nm}$ sized $\mathrm{SiO}_{2}$ nanoparticles were obtained using a modified Stöber method. ${ }^{52}$ In a typical synthesis, $35.7 \mathrm{~mL}$ of absolute ethanol was mixed with 5 $\mathrm{mL}$ water and $0.8 \mathrm{~mL}$ of ammonia and stirred for 5 10 minutes at room temperature. Then $1 \mathrm{~mL}$ of TEOS and $1 \mathrm{~mL}$ of APTES-FITC were added and the mixture was allowed to react at room temperature for $1 \mathrm{~h}$. Afterward, $\mathrm{SiO}_{2}$ nanoparticles were washed with water and ethanol and suspended in $20 \mathrm{~mL}$ of water. To prepare hollow mesoporous silica nanoparticles,${ }^{53}$ CTAB (2 g) and TEA (20 mg) were dissolved in $20 \mathrm{~mL}$ of DI water and stirred at room temperature for $1 \mathrm{~h}$. Then, $10 \mathrm{~mL}$ of the previous $\mathrm{SiO}_{2}$ suspension was added and stirred at room temperature for $1 \mathrm{~h}$ before addition of $0.15 \mathrm{~mL}$ of TEOS. The mixture was stirred for $1 \mathrm{~h}$ at $80^{\circ} \mathrm{C}$ in a water bath and the mixture was then cooled down to $50^{\circ} \mathrm{C}$ followed by addition of $636 \mathrm{mg}$ of sodium carbonate $\left(\mathrm{Na}_{2} \mathrm{CO}_{3}\right)$, under constant stirring for $30 \mathrm{~min}$. To remove the CTAB, the product was extracted for $24 \mathrm{~h}$ with a $1 \mathrm{wt} \%$ solution of $\mathrm{NaCl}$ in methanol at room temperature. This process was carried out for at least 3 times to ensure complete removal of CTAB. For PEG grafting, $8 \mathrm{mg}$ of the recovered particles were dispersed in $50 \mathrm{~mL}$ of DMSO and then $500 \mu \mathrm{L}$ of APTES were added. After the mixture was stirred for 20 hours, amine-functionalized HSNPs $\left(\mathrm{HSNP}-\mathrm{NH}_{2}\right)$ were recovered by centrifugation and washed with ethanol twice. Next, HSNP-NH $\mathrm{NH}_{2}$ particles were re-dispersed in $5 \mathrm{~mL}$ of DMSO, and $8 \mathrm{mg}$ of NHS-PEG $5000-\mathrm{MAL}$ was added. The mixture was then stirred at room temperature for 2 hours under anhydrous conditions before being harvested by centrifugation. 


\section{Synthesis of PEGylated Double-layered PEI-Silica Nanoparticles (PSNPs). For}

the synthesis of $\mathrm{SiO}_{2} @ \mathrm{PEI}, 20 \mathrm{~mL}$ of a suspension of $100 \mathrm{~nm}$ FITC-doped silica nanoparticles (vide supra) was added dropwise to the same volume of an aqueous solution of PEI $\left(10 \mathrm{mg} \cdot \mathrm{mL}^{-1}\right){ }^{54}$ After 3 additional hours of mixing, particles were recovered by centrifugation, washed 2 times in DI water and finally re-suspended in DI water. For the synthesis of $\mathrm{SiO}_{2} @ \mathrm{PEI} @ \mathrm{SiO}_{2},{ }^{47} 8 \mathrm{~mL}$ of the $\mathrm{SiO}_{2} @ \mathrm{PEI}$ solution was mixed with $5 \mathrm{~mL}$ absolute ethanol and $0.8 \mathrm{~mL}$ of ammonia and stirred for 10 minutes at room temperature. Then $0.2 \mathrm{~mL}$ of TEOS and $20 \mu \mathrm{L}$ APTES-FITC was added and the mixture was allowed to react at room temperature for $16 \mathrm{~h}$. Afterward, $\mathrm{SiO}_{2} @ \mathrm{PEI} @ \mathrm{SiO}_{2}$ nanoparticles were washed with water and ethanol and suspended in $20 \mathrm{~mL}$ of water. For the deposition of the second PEI and silica layers, the synthetic procedure was similar to the previous one, except for the final addition of $200 \mu \mathrm{L}$ APTES to obtain $\mathrm{SiO}_{2} @ \mathrm{PEI} @ \mathrm{SiO}_{2} @ \mathrm{PEI} @ \mathrm{SiO}_{2}-\mathrm{NH}_{2}$ nanoparticles. Next, these particles were re-dispersed in $5 \mathrm{~mL}$ of $\mathrm{DMSO}$, and $8 \mathrm{mg}$ of $\mathrm{NHS}^{-P_{E G}}{ }_{5000}-\mathrm{MAL}$ was added. The mixture was then stirred at room temperature for 2 hours under anhydrous conditions before harvesting by centrifugation.

Nanoparticle Characterization. Particle size distribution, polydispersity index and zeta potential $(\zeta)$ were measured in DI water or cell culture medium (DMEM) using a ZetaSizer Nano (Malvern Instruments Ltd., Worcestershire, UK). Particles were also imaged using Transmission Electron Microscopy (TEM) on a FEI Tecnai $F_{20}$ U-TWIN electron microscope (TEM) (FEI Company, Philips, Netherlands). Dynamic Light 
Scattering (DLS) was used to determine the hydrodynamic diameter of the nanoparticles in Milli-Q water or in culture medium. The reading was carried out at an angle of $90^{\circ}$ to the incident beam $(632 \mathrm{~nm})$. The Contin algorithm was used to analyze the autocorrelation functions. Fluorescence spectra of the particles suspended in water were recorded on a Horiba Jobin Yvon fluorescence spectrometer with an excitation wavelength of $485 \mathrm{~nm}$. Nitrogen $\left(\mathrm{N}_{2}\right)$ sorption measurements were performed at $77 \mathrm{~K}$ using an accelerated surface area and porosimetry analyzer with Brunauer-Emmett-Teller (BET) calculations for the surface area.

Dissolution and Degradation Assays in Buffer and Culture Medium. To carry out the in vitro degradation experiments in abiotic conditions, a suspension of each type of PEGylated particles at a $0.4 \mathrm{mg} \cdot \mathrm{mL}^{-1}$ concentration was left under mild stirring at $37^{\circ} \mathrm{C}$ in phosphate buffer saline (PBS) $1 \mathrm{X}$ or DMEM. The fluorescence intensity of the medium was analyzed at selected time intervals after centrifugation in a Nanosep® $3 \mathrm{kD}$ centrifugal device to separate particles, that were further used for TEM observation, and solutions to monitor the proportion of released silane-FITC over time. ${ }^{39}$ All experiments were performed in triplicate.

Cells and Treatments with Nanoparticles. Normal human dermal fibroblasts (from Promocell) were grown in DMEM supplemented with FCS (10\%), penicillin (100 units.mL $\left.{ }^{-1}\right)$, streptomycin (100 mg.mL $L^{-1}$, from Gibco BRL) and fungizone $\left(0.25 \mathrm{mg} . \mathrm{mL}^{-1}\right.$, from Gibco BRL). The culture flasks $\left(75 \mathrm{~cm}^{2}\right)$ were kept at $37{ }^{\circ} \mathrm{C}$ in $95 \%$ humidity and $5 \% \mathrm{CO}_{2}$ atmosphere. At confluence, fibroblasts were removed from cultured flasks by 
treatment with $0.1 \%$ trypsin and $0.02 \%$ EDTA. Cells were rinsed, centrifuged and dispersed in the supplemented DMEM medium. Fibroblasts were used at passage 8 for the experiments.

Nanoparticle Internalization, Degradation and Release. The cells were seeded at a density of 30,000 cells per well in 24 -well plate with round glass coverslips at the bottom of the wells and kept $24 \mathrm{~h}$ with culture medium at $37{ }^{\circ} \mathrm{C}$ in $95 \%$ humidity and $5 \%$ $\mathrm{CO}_{2}$ atmosphere previous to incubation with $0.4 \mathrm{mg} \cdot \mathrm{mL}^{-1}$ of the three types of silica nanoparticles. For fluorescence microscopy, cells were collected at day 1, 2 and 7, washed three times with PBS 1X, and fixed with $4 \%$ paraformaldehyde in PBS $(1 \mathrm{~h}, 4$ ${ }^{\circ} \mathrm{C}$ ). Staining of the nuclei with DAPI (Invitrogen) was perform via incubation for $15 \mathrm{~min}$ at room temperature and rinsing with HBSS $1 \mathrm{X}$ before observation with a fluorescent microscope (Axio 100, Carl Zeiss). For TEM, cells were fixed at day 1, 2 and 7 using $3.63 \%$ glutaraldehyde in a $0.05 \mathrm{M}$ sodium cacodylate buffer with $0.3 \mathrm{M}$ saccharose for 1 h at $4{ }^{\circ} \mathrm{C}$. Samples were washed three times before post-fixing with $2 \%$ osmium tetraoxide for $1 \mathrm{~h}$ at $4{ }^{\circ} \mathrm{C}$. Cells were then detached from the culture flasks by scratching and centrifuged. The pellets were embedded in a $4 \mathrm{mg} \cdot \mathrm{mL}^{-1}$ collagen hydrogel to ease sectioning and cellular observations. With this purpose, $50 \mu \mathrm{L}$ of the type I collagen solution (in $17 \mathrm{mM}$ acetic acid) was neutralized by $5 \mu \mathrm{L}$ of PBS and mixed with the pellet. Mixtures were left for 30 minutes at $20^{\circ} \mathrm{C}$ to allow collagen gelation. Recovered hydrogels were fixed with $4 \%$ paraformaldehyde in PBS $\left(1 \mathrm{~h}, 4{ }^{\circ} \mathrm{C}\right)$. Last, cell-containing collagen hydrogels were dehydrated with an ascending ethanol series ending with 
propylene oxide and embedded in araldite. Ultrathin sections were prepared with an Ultracut ultramicrotome (Leica, France). Slides were analyzed with a JEOL 1011 electron microscope operating at $120 \mathrm{kV}$. Images were obtained for at least 10 cells for each sample.

To follow the kinetics of particles degradation and release, continuous cultures were first performed over 1 week in the presence of $0.4 \mathrm{mg} \cdot \mathrm{mL}^{-1}$ silica nanoparticles. After this delay, the culture medium was removed and cells were rinsed before adding fresh medium and performing an additional incubation period of 7 days. Fluorescence and TEM imaging, as well as fluorescence measurements of the whole medium and of its soluble content were performed as described above. All experiments were performed in triplicate.

Toxicological Assays. Cellular metabolic activity of cells in 24-well plate after 2 days of contact with silica particles at concentrations $0.025-0.400 \mathrm{mg} \cdot \mathrm{mL}^{-1}$ and after 1,2 , 7 and 14 days of incubation with $0.4 \mathrm{mg} \cdot \mathrm{mL}^{-1}$ silica nanoparticles was evaluated using Alamar Blue assay $(n=6)$. After incubation, cells were rinsed with medium and incubated at $37{ }^{\circ} \mathrm{C}$ in a humidified $5 \% \mathrm{CO}_{2}$ air atmosphere for $4 \mathrm{~h}$ with a $10 \%$ solution of Alamar blue in phenol red-free culture medium. Absorbance of the medium at $570 \mathrm{~nm}$ and 600 $\mathrm{nm}$ was recorded with a UV-visible spectrophotometer and the percentage of dye reduction was calculated. The results were expressed as a percentage of viability assessed 


\begin{abstract}
in control samples (incubated with no nanoparticles). It is worth mentioning that incubation of the particles alone with Alamar Blue gave negligible absorbance values.
\end{abstract}

Statistical Analysis. Statistical significance of differences was evaluated by a Wilcoxon-MannWhitney test. This test was chosen instead of Student t test according to the result of Shapiro-Wilk normality test. A value of $\mathrm{P}<0.05$ was considered significant.

\title{
RESULTS AND DISCUSSION
}

\section{Synthesis and Functionalization of Nanostructured Silica Particles. Silica} nanoparticles with different internal structures were synthesized and characterized using transmission electron microscopy (TEM), dynamic light scattering (DLS) and zeta potential measurements. PEGylated BSA-coated silica nanoparticles, PEI-coated silica nanoparticles and hollow mesoporous silica nanoparticles were named as BSNPs, PSNPs and HSNPs respectively.

Fairly uniform BSNPs were prepared by using a modified Stöber method in the presence of BSA. As shown in the TEM images (Figure 1a,d), the BSNPs are spherical in shape with a mean diameter of $\sim 45 \mathrm{~nm}$. They exhibit a well-contrasted core-shell structure suggesting that they consist of a silica-rich layer coating a BSA-rich internal domain. ${ }^{50}$ 


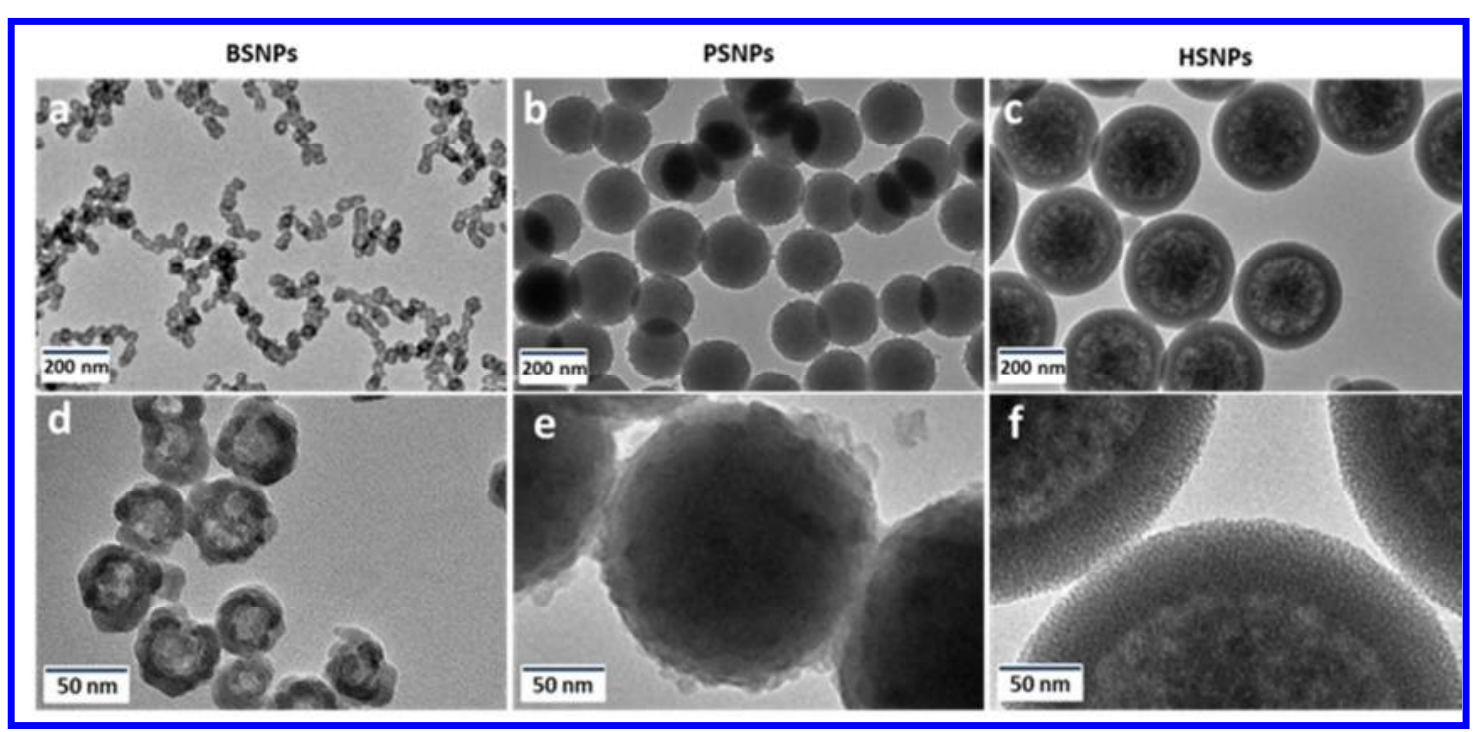

Figure 1 : TEM images of the as-prepared PEGylated fluorescent silica nanoparticles: (a, d) BSNPs; (b, e) PSNPs and (e, f) HSNPs.

Table 1 : Mean diameter $D_{m}$ from DLS and TEM, Zeta potential $\zeta$ in deionized water and culture medium.

\begin{tabular}{lllll}
\hline & $D_{m}$ DLS & $D_{m}$ TEM & $\zeta$ water & $\zeta$ medium \\
& $(\mathrm{nm})$ & $(\mathrm{nm})$ & $(\mathrm{mV})$ & $(\mathrm{mV})$ \\
\hline BSNPs & $109 \pm 6$ & $44 \pm 2$ & $-15 \pm 1$ & $-8 \pm 1$ \\
PSNPs & $190 \pm 6$ & $176 \pm 4$ & $-8 \pm 1$ & $-5 \pm 1$ \\
HSNPs & $310 \pm 10$ & $261 \pm 7$ & $-11 \pm 1$ & $-7 \pm 1$ \\
\hline
\end{tabular}

DLS measurements in deionized water indicated a two-fold increase in the mean diameter $D_{m}$, compared to TEM, suggesting that some aggregation occurred, although to a limited 
extent (Table 1 and Figure S1). This aggregation was confirmed by performing a DLS analysis of a BSNPs suspension just after sonication, yielding to a $\mathrm{D}_{m}$ value of $44 \pm 10$ $\mathrm{nm}$ (Figure S1). The successful functionalization process with amine and then PEG groups was evidenced by size and $\zeta$ potential modifications (Table S1 in ESI): whereas the as-prepared BSA@ $\mathrm{SiO}_{2}$ exhibited a strongly negative $\zeta$ value, APTES grafting led to a slightly positive $\zeta$ value due to ammonium groups. This was correlated with an increase in the $D_{m}$ value as obtained by DLS, in agreement with the decrease of $\zeta$ absolute value leading to a lower colloidal stability. After PEG grafting, the $D_{m}$ value obtained by DLS decreased, due to the ability of the polymer coating to limit BSNPs aggregation, and the $\zeta$ value became negative, due to the reaction of some of the surface amine groups with NHS-PEG ${ }_{5000}-\mathrm{MAL}$.

PSNPs were obtained from plain silica nanoparticles by a layer-by-layer method using branched PEI $(25 \mathrm{kDa})$. Starting from $\sim 100 \mathrm{~nm}$ Stöber nanoparticles, two successive sequences of PEI deposition/silica coating were performed. Variations in $\zeta$ value occurred as expected, i.e. $\zeta>0$ for outer PEI layer and $\zeta<0$ after silica deposition (Table S1 in ESI). Again, APTES grafting turned the particle surface positive while PEG conjugation ultimately led to negatively-charged PSNPs in deionized water (Table 1). The accompanying modifications of hydrodynamic diameter, as obtained by DLS, are difficult to analyze in details in terms of size variations as they are the result of PEI conformation on the particle surface, silica layer thickness as well of the aggregation tendency of the different systems. Nevertheless TEM imaging (Figure 1b) provides a $D_{m}$ 
value of $\sim 175 \mathrm{~nm}$ and therefore an overall coating thickness of $\sim 35 \mathrm{~nm}$, clearly evidenced at higher magnification (Figure 1e).

Hollow HSNPs were prepared by etching $100 \mathrm{~nm}$-Stöber nanoparticles following the method proposed by Chen et al. ${ }^{53}$ TEM imaging clearly showed that HSNPs have a spherical morphology and uniform particle size $\sim 260 \mathrm{~nm}$, with a porous shell thickness of $\sim 50 \mathrm{~nm}$ and a highly porous core, although a central denser region, corresponding to unreacted part of the starting Stöber particles, could sometimes be distinguished (Figure 1c,f). The overall synthetic process was also followed by DLS and $\zeta$-metry (Table S1), showing that despite the fact that APTES-modified intermediates are particularly prone to aggregation $\left(D_{m}=780 \pm 40 \mathrm{~nm}\right)$, the PEG coating efficiently enhances the colloidal stability of final HSNPs particles $\left(D_{m}=310 \pm 10 \mathrm{~nm}\right.$ in deionized water compared to 260 $\pm 7 \mathrm{~nm}$ from TEM) (Table 1). Nitrogen adsorption-desorption isotherm measurements indicated that the HSNPs had a relatively high specific surface area of $c a .550 \mathrm{~m}^{2} \cdot \mathrm{g}^{-1}$ and a well-defined mean pore size of $3.9 \mathrm{~nm}$ (Figure S2).

Altogether, when the three types of particles are compared, their size, as obtained from both DLS in deionized water and TEM, vary in the order HSNPs > PSNPs > BSNPs. In terms of surface properties, their zeta potential in deionized water ( $\mathrm{pH} 6.5$ ) is always negative and follows the PSNPs $>$ HSNPs $>$ BSNPs evolution. Interestingly, the same order of $\zeta$ values is found for $\mathrm{NH}_{2}$-grafted particles, suggesting that the PEG coupling occurred in a similar extent for the three systems. This order is preserved over 
the whole $\mathrm{pH}$ 2-11 range with apparent isoelectric points (IEP) of ca. 4.5, 5.5 and 6.2 for BSNPs, HSNPs and PSNPs, respectively (Figure S3). It is also maintained in cell culture medium although with some minor variations between the different particles. A systematic increase in $\zeta$ values compared to deionized water is observed that very likely indicates the surface adsorption of positively-charged proteins present in FCS-enriched DMEM (Table 1). ${ }^{55}$ The incorporation of FITC within the different nanoparticles was checked by fluorescence measurements. As shown in Figure S4, the FITC-doped silica nanoparticles gave rise to a strong emission, with no significant shift in the maximum wavelength compared to the dye alone. However, at a constant particle concentration, the signal intensity varied from one particle type to the other. Such a difference may reflect various silica contents in the nanoparticle structures. For instance, the lowest intensity was obtained for BSNPs, i.e. hybrid nanoparticles containing large amounts of BSA. However, other processes such as FITC fluorescence variations due to different dye environment and mobility may also contribute to these differences. ${ }^{56}$

\section{Behaviour of Nanoparticles in Buffer and Cell Culture Medium. To investigate}

the influence of the internal structure of silica nanoparticles on their degradation in abiotic (i.e cell-free) media, TEM imaging and monitoring of the kinetics of silica dissolution was first performed in a PBS buffer $(\mathrm{pH} 7.4)$ at $37{ }^{\circ} \mathrm{C}$ over 2 weeks. After one week, all nanoparticles showed a clear tendency to form aggregates under TEM imaging conditions (Figure 2). For BSNPs, the core-shell structure is overall preserved but some particles appear to have grown in size and the contrast between the inside and outside 
parts has increased compared to the initial particles. For PSNPs, the outer silica shell is less well-defined. No further evolution could be evidenced for these two sets of particles after one additional week, except for a higher tendency to aggregate. In parallel, the internal structure of HSNPs does not appear much modified after one week but some deformed particles are observed. After two weeks, they have turned into highly porous particles with a weaker contrast between the shell and the core parts, some of them being obviously fractured.

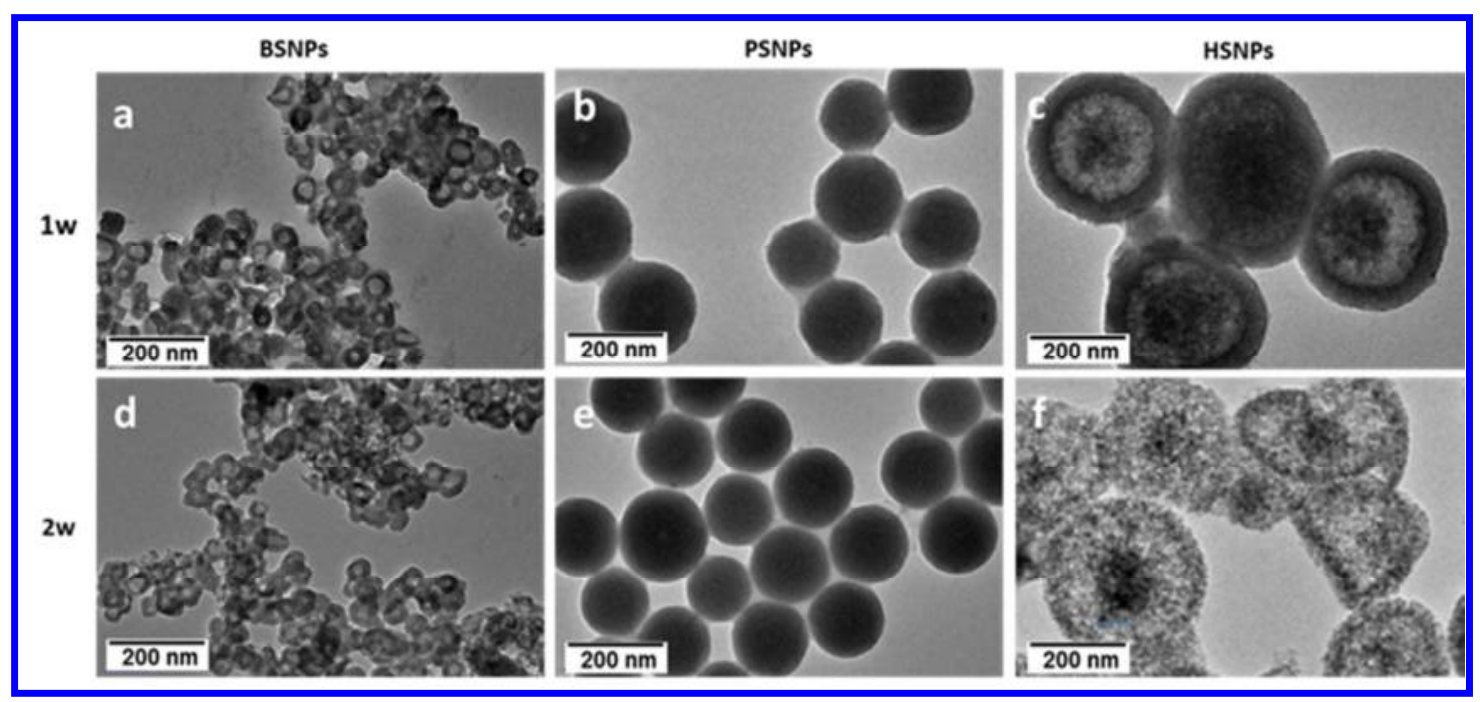

Figure 2: TEM imaging of the structural evolution of silica nanoparticles in PBS after (a-c) one and (d-f) two weeks.

The dissolution of these different particles was also studied in the DMEM cell culture medium at $37{ }^{\circ} \mathrm{C}$ (Figure $\mathbf{3}$ and Figure $\mathbf{S 5}$ for higher magnification images). For BSNPs, after one week, some particles have grown in size while others are broken. Only small fragments could be recovered after 2 weeks. For PSNP samples after 1 week, the 
outer coating has a shallow appearance and is decorated with porous particles 10-30 nm in diameter. Strikingly, after two weeks, two populations of particles are observed: larger ones, $c a .100-150 \mathrm{~nm}$ in diameter, exhibiting a dense core and a porous shell, and smaller ones, 20-50 $\mathrm{nm}$ in size, with a porous internal structure resembling that of previously-observed decorating particles. Such an evolution would suggest dissolution of the silica material constituting PSNPs shell and core and its reprecipitation into smaller particles. To support this hypothesis, a more detailed analysis by TEM of the kinetics of degradation of these nanoparticles was performed, evidencing that the progressive appearance of the porous external small particles was correlated with a decrease in the density of the largest ones (Figure S6)

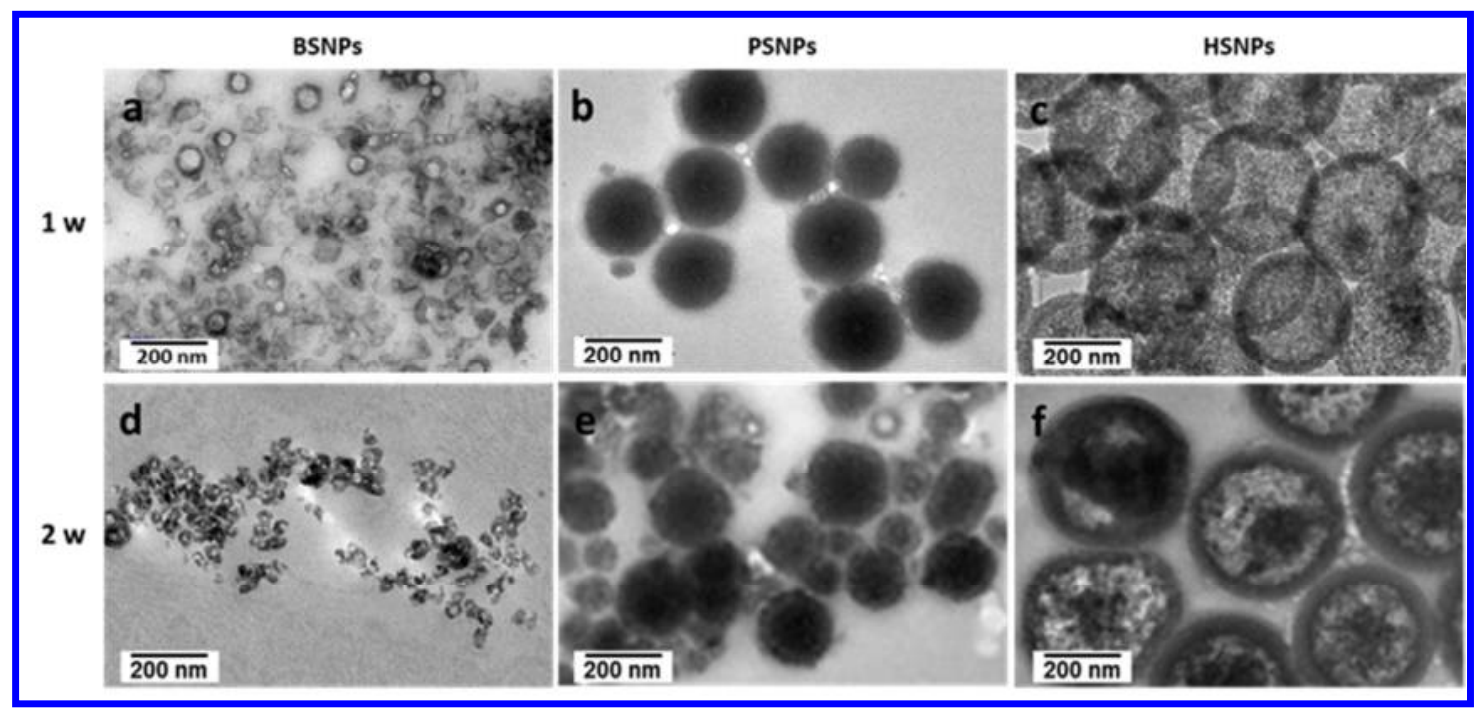

Figure 3 : TEM imaging of the structural evolution of silica nanoparticles in DMEM culture medium after (a-c) on week and (d-f) two weeks.

For HSNPs, TEM images after 1 week are highly reminiscent of those previously obtained in PBS after 2 weeks. Within two weeks, after centrifugation and washing, only 
a few of these nanoparticles could be recovered with an intact morphology and imaged. These particles seem to have grown in size and the shell and core particle appear denser and separated by a highly porous, if not empty, region. Hence, overall, the culture medium appears to speed up the degradation process of all particles compared to PBS at a similar $\mathrm{pH}$.

In parallel, the dissolution rate of the particles in culture medium was investigated through the monitoring of the fluorescence of the solution after centrifugation and filtration. Because these steps eliminate silica particles and since FITC forms a stable covalent bond with the silica network, the fluorescence signal can only correspond to FITC-silane or soluble FITC-polysiloxane molecules released upon silica dissolution. ${ }^{39}$

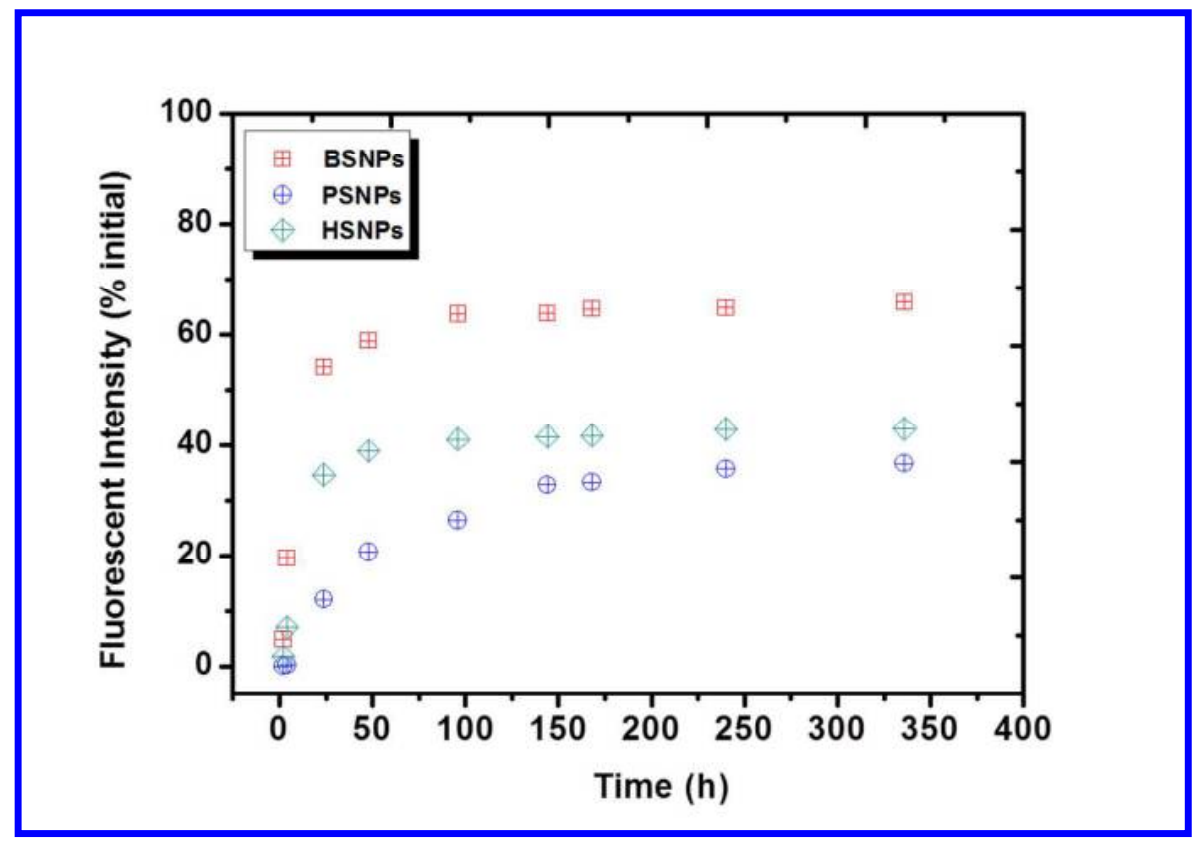


Figure 4 : Evolution of the content of the culture medium over 2 weeks in the presence of silica nanoparticles as monitored by the fluorescence intensity of FITC-labelled soluble forms of silica.

After 1 day, more than $50 \%$ of the BSNPs particles are dissolved and the maximum dissolution of $65 \%$ is reached after 1 week (Figure 4). For HSNPs, a similar rapid dissolution is observed but the maximum dissolution of $40 \%$ is reached after 1 week. PNSPs dissolve much more slowly (ca. $10 \%$ after 1 day) and, after 2 weeks, only $30 \%$ of the initial fluorescent silanes are present in a soluble form. These data confirm the low stability of BSNPs in DMEM. For PNSPs, the low degradation rate and extent are in favor of the proposed mechanism of progressive reprecipitation of the soluble forms originating from the shell and then core particle dissolution. HSNPs represent an intermediate case, with fast initial dissolution rate followed by a plateau and low dissolution extent, suggesting that some reprecipitation reactions also occur, but in a less efficient manner than in PNSPs

Nanoparticles Behaviour in the Presence of Fibroblasts. To study the possible impact of nanoparticle structure and degradation on cell viability, NHDF cells were used as model organisms and the Alamar Blue method, which measures the mitochondrial activity of the cells, was selected to monitor the cellular response to nanoparticles (Figure 5). After a $48 \mathrm{~h}$ incubation period, in all the range of concentrations tested, BSNPs and HSNPs did not show a significant toxicity while PSNPs induced an initial 
slight reduction of cell viability in all conditions, down to $75 \%$ of cell viability at a 400 $\mu \mathrm{g} . \mathrm{mL}^{-1}$ dose. However, at this concentration, NHDFs recovered from PSNPs cytotoxicity after 1 week.

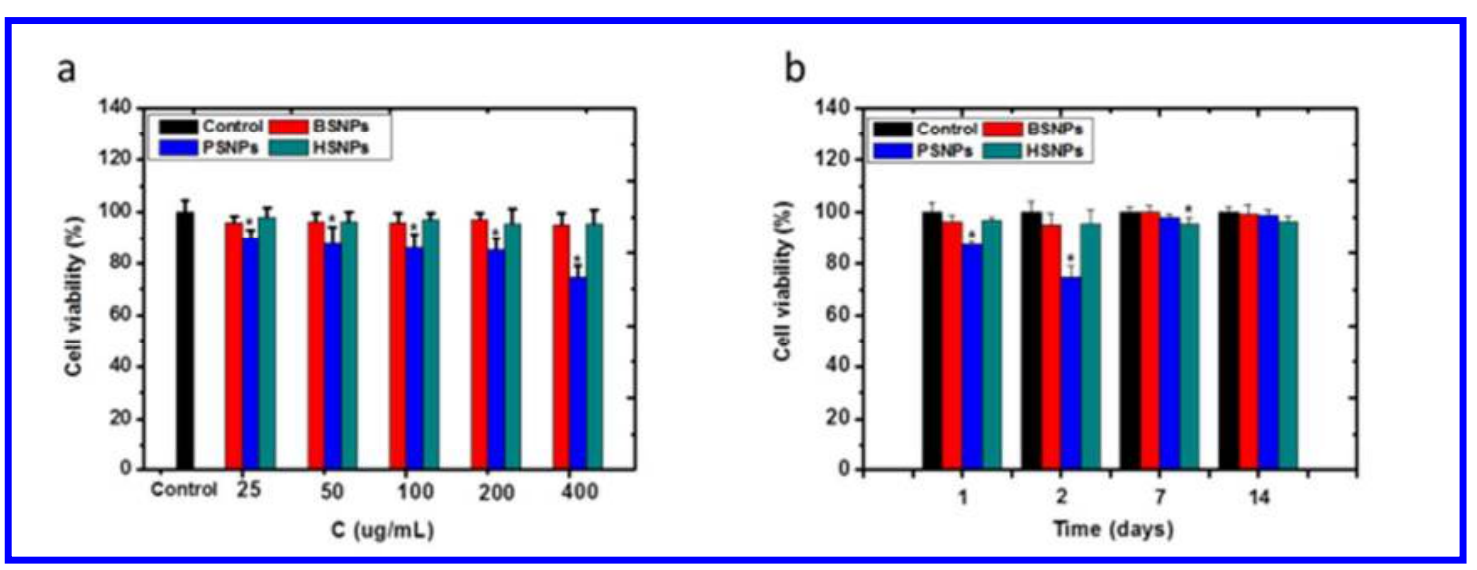

Figure 5: (a) Alamar blue assay results for NHDF cells after incubation with silica nanoparticles at various concentrations $\left(25-400 \mu \mathrm{g} \cdot \mathrm{mL}^{-1}\right)$ for 2 days. (b) Alamar blue assay results for NHDF cells after incubation with $400 \mu \mathrm{g} \cdot \mathrm{mL}^{-1}$ of silica nanoparticles at various time intervals (1-14 days). The data represent six separate experiments. Mean values $\pm \mathrm{SD} . * \mathrm{P}<0.05$ versus a control group.

Referring to the dissolution kinetics shown in Figure 4, neither BNSPs nor their degradation products have significant cytotoxic effects. PNSPs are cytotoxic on the short term, a period where the outer layers are slowly dissolving. This result can be correlated to previous reports showing that the presence of an external silica layer decreases the toxicity of poly-lysine-coated gold nanoparticles. ${ }^{57}$ On this basis, it can be suggested that the initial dissolution of the silica layers leads to the release of cytotoxic PEI. However, 
the redeposition of smaller silica particles on PNSPs surface, as observed in Figure 3, may circumvent this effect on the longer term.

The particle uptake by NHDFs in culture medium was followed by fluorescent microscopy using the same silica concentration $\left(400 \mu \mathrm{g} \cdot \mathrm{mL}^{-1}\right)$. After $24 \mathrm{~h}$, silica nanoparticles associated with cells were evidenced but many of them appeared to be attached to the cell membrane as aggregates (Figure S7). With time, these aggregates became less visible and the fluorescent signal appeared confined within the cell interior, with a higher intensity for PSNPs and HSNPs compared to BSNPs after 2 weeks (Figure 6).

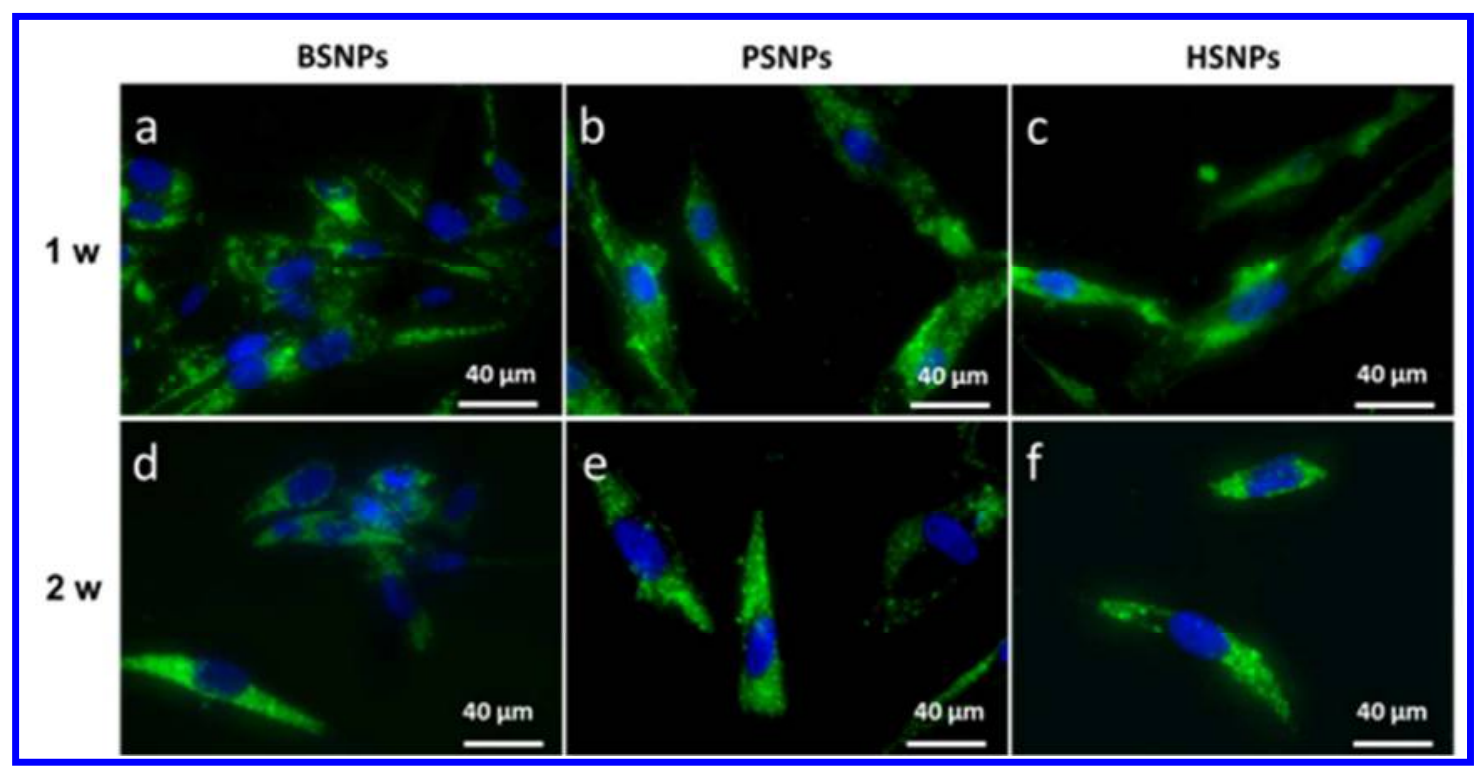


Figure 6: Fluorescence optical imaging of human dermal fibroblast cells after (a-c) 7 days and (d-f) 14 days of contact with $400 \mu \mathrm{g} \cdot \mathrm{mL}^{-1}$ silica nanoparticles. DAPI was used for blue staining of the nucleus and green fluorescence corresponds to FITC. (Scale bar $=$ $40 \mu \mathrm{m})$.

However, to fully ascertain particle internalization, TEM imaging was performed (Figure 7 and Figure S8 for lower magnification images). After 1 week, BSNPs are sparingly found as intracellular aggregates of particles that have decreased in size as an apparent consequence of shrinking after core material removal. PSNPSs and HSNPs are more easily distinguished both outside and inside the cells. The former appear as plain particles decorated with smaller particles. For HSNPs the shell has grown in thickness while the internal structure has started to degrade, as previously observed in DMEM after 2 weeks. 


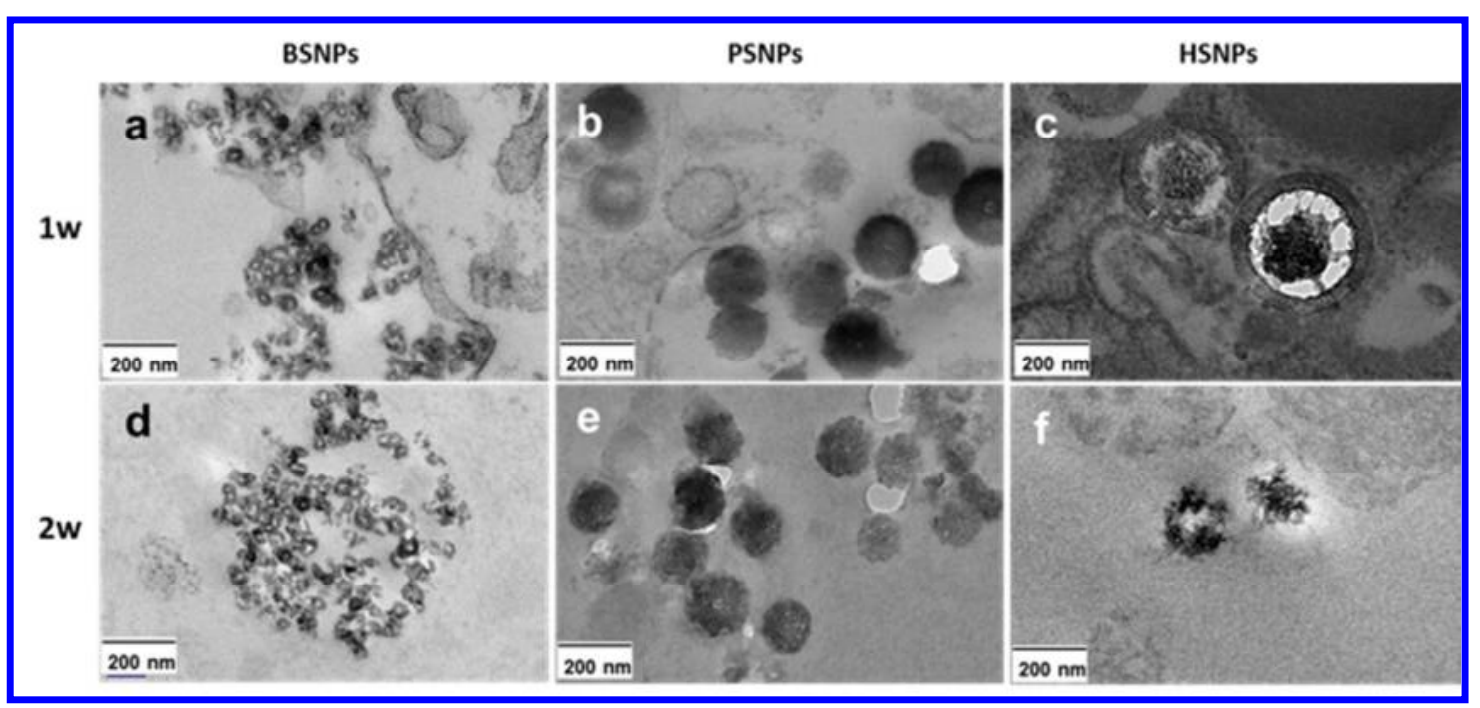

Figure 7 : TEM images of NHDF cells after (a-c) 1 week and (d-f) 2 weeks of contact with $400 \mu \mathrm{g} \cdot \mathrm{mL}^{-1}$ silica nanoparticles.

Degradation and Release Kinetics. The kinetics of degradation and release of the nanoparticles was further studied by keeping NHDFs in contact with silica nanoparticles for 1 week after which the cells were rinsed off the remaining external particles and incubated in fresh culture medium for an additional week. Over the first $24 \mathrm{~h}$, for all particles, the total fluorescence of the solution is initially very low and then increase progressively up to $c a .80 \%$ of the intensity of the initial particle suspension (Figure 8a). This can be correlated with fluorescence optical imaging indicating that particles first adhere on the cell surface (decreasing the amount of free particles in solution) and are then progressively detached. Yet a decrease in total fluorescence intensity is observed after $48 \mathrm{~h}$, that may reflect enhanced particle internalization. ${ }^{39}$ Considering the release of soluble forms in the culture medium, the kinetics profiles are very similar to those obtained in the absence of NHDF. However, the maximum apparent dissolution ratio is 
significantly smaller for BSNPs (50\% compared to $60 \%$ without NHDF) and PSNPs (20 $\%$ compared to $30 \%$ ). This would suggest that a fraction of the soluble forms is retained by the cells.
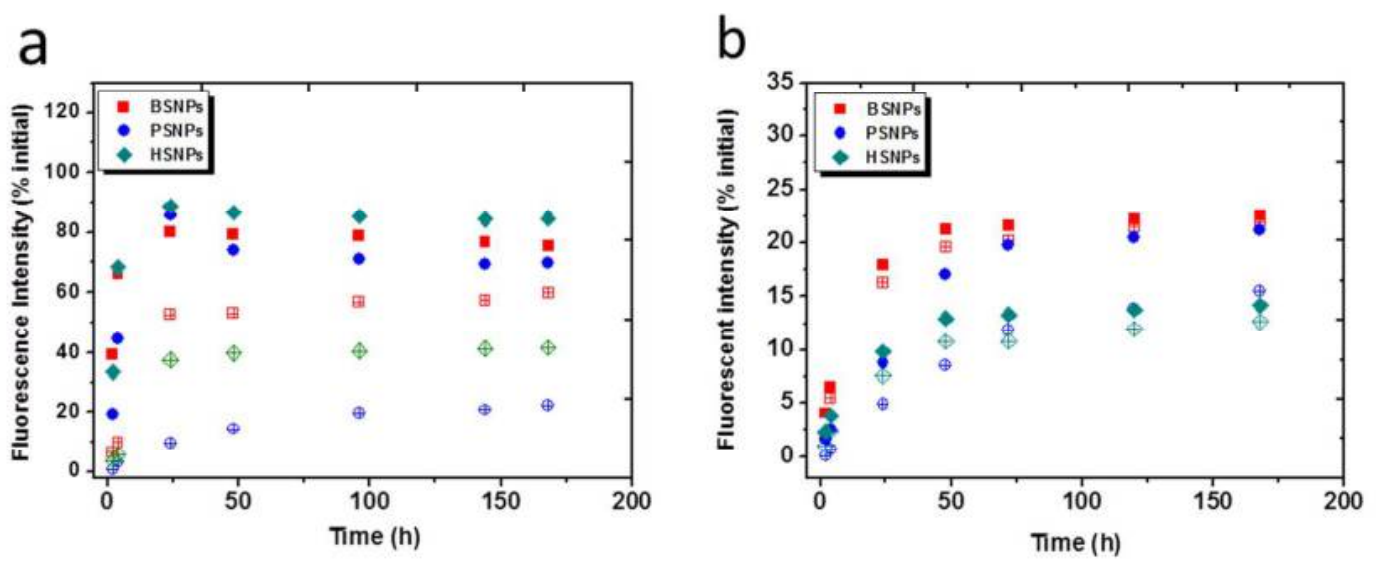

Figure 8: Evolution of the content of the NHDF cells culture medium as monitored by the fluorescence intensity of FITC-labelled condensed and soluble forms of silica (filled symbols) and soluble forms only (open symbols) (a) over 1 week in the presence of silica nanoparticles and (b) over 1 additional week in a fresh medium.

After cell rinsing and incubation in a fresh, particle-free medium for an additional week, intracellular nanoparticles were more difficult to observe in TEM imaging fields (Figure 7d and Figure S8). When this was possible, we did not evidence any clear change for BSNPs compared to day 7. PSNPs have significantly decreased in size and exhibit a porous structure. No well-defined HSNPs could be observed after this period and only fragments could be imaged that may originate from their full dissociation. In parallel, the total FITC fluorescence intensity in the culture medium, initially equal to 
zero because of the incubation in a fresh medium, increased with time (Figure 8b). At the end of this additional period, almost all the initial fluorescence intensity (as calculated by adding 1 week values obtained from Figure $8 \mathrm{a}$ and 2 weeks values from Figure $8 \mathrm{~b}$ ) was recovered for the three kinds of particles, indicating that a negligible fraction of silica remains inside the cells, in agreement with TEM experiments. However, whereas BSNPs and HSNPs were almost fully released in a soluble form, more than $30 \%$ of PSNPs were externalized in a colloidal form.

Degradation Pathways. BSNPs initially show TEM-contrasting internal features that suggest that they consist of a protein-rich interior surrounded by a silica-rich coating. In PBS, these particles grow in size, with an apparent leakage of the core material, the process being accelerated in culture medium. Finally, particle degradation occurs, leaving much smaller empty particles. A similar process seems to occur within the NHDF cells, although at a higher rate. Additional experiments performed using BSNPs incorporating FITC-labelled BSA showed that, after one week of contact with NHDF, the protein was hardly detectable within or at the vicinity of the cells whereas silica was still present in large amounts (compare Figure S9 with Figure 6a). The most likely explanation of these evolutions is that water penetration within the particle leads to the swelling of the BSA core, disruption of the silica shell and leakage of the protein (Figure 9). As suggested earlier, the enzymatic degradation of BSA in the intracellular compartment may contribute to accelerate these processes. 

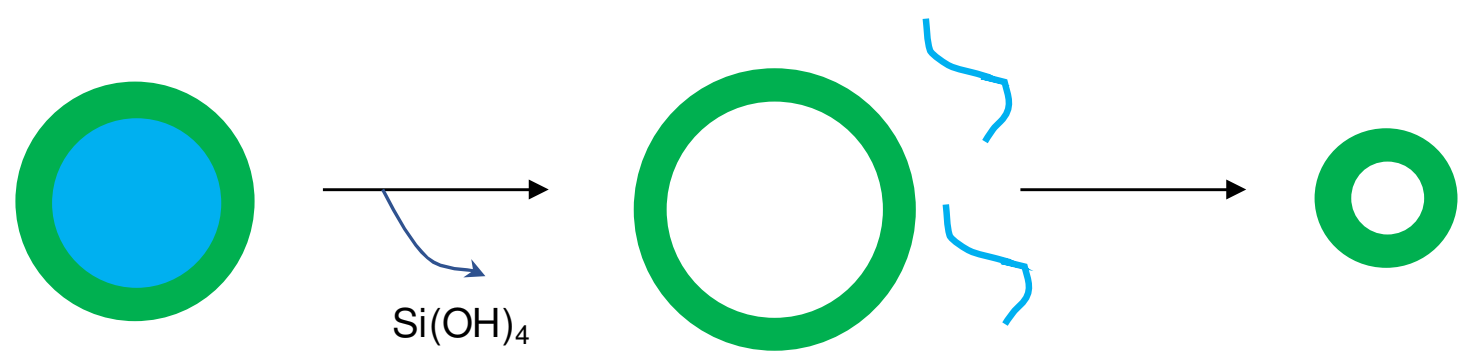

\section{BSNPs}

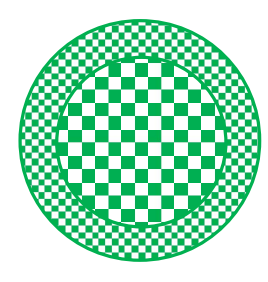

HSNPs
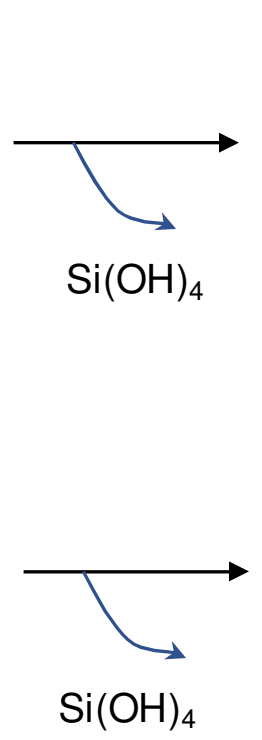

PSNPs

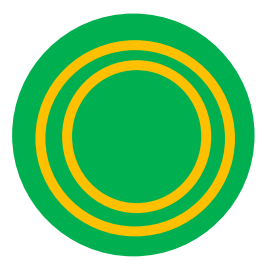

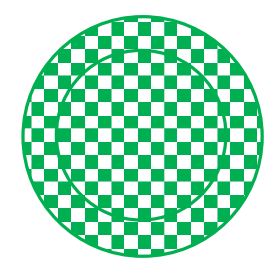
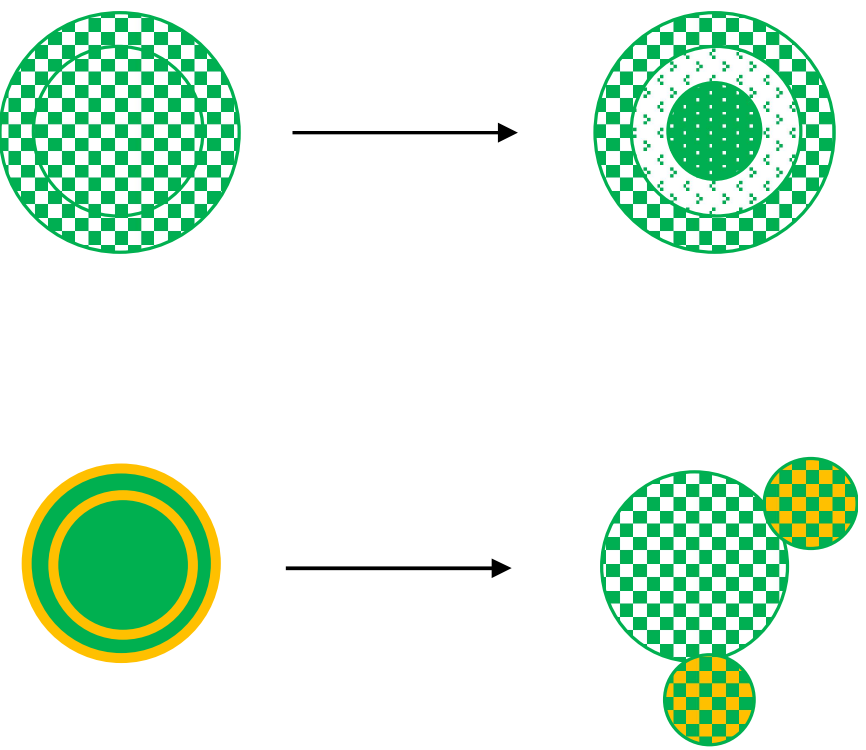

\section{Dense silica}
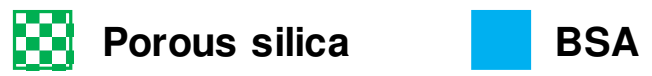

PEI

Figure 9: Schematic representation of the degradation pathways of BSNPs, HSNPs and PSNPs in

DMEM culture medium.

For HSNPs, silica dissolution in PBS apparently occurs first from the shell, resulting in particles that are almost uniformly porous except for the central part and are prone to 
deformation and breaking (Figure 9). The same process occurs in culture medium but after two weeks, the only remaining particles exhibit a highly contrasted shell and a denser central part, separated by a nearly empty corona. Such structures were also observed after one week within cells and evolved towards a complete disintegration after 2 weeks. While the initial shell dissolution should be favored by its mesoporous structure, the following process can be explained considering the dissolution of the more porous (i.e. more soluble) silica corona located between the shell and the core particle and its reprecipitation on these two denser (i.e. less soluble) regions, based on the Ostwald ripening principle.

Overall BSNPs and HSNPs evolve more rapidly but along similar pathways in PBS, in DMEM and in the intracellular space. When experiments in buffer and culture medium are compared, $\mathrm{pH}$ and total silica concentration are similar. Ionic strength of DMEM is $0.13 \mathrm{M}$, being therefore comparable with the $0.16 \mathrm{M}$ value for PBS 1X. However, DMEM supplemented with FCS contains several bio-organic components (amino acids, proteins) that can interact with silica. For instance, it was shown that histidine and phenylalanine could promote silica dissolution. ${ }^{28}$ Extending this discussion to intracellular compartments is indeed difficult as their composition is complex and variable. Nevertheless it is worth pointing out that lysozomes were reported to contain a high fraction of free amino acids. ${ }^{58}$ It is also interesting to note that intracellular compartments that are involved in nanoparticle trafficking are reported to be slightly (endosomes) to significantly (lysozomes) acidic so that a decrease of silica dissolution 
rate could be expected compared to neutral conditions of PBS/DMEM medium. While this point would require a detailed investigation of the particle structural modification in the first hours of the internalization process, our experiments suggest that the difference in $\mathrm{pH}$ conditions is not of primary importance for their long-term fate.

However, another important point must be taken into consideration. The full release of silicon species from the cells to the medium clearly demonstrates that the products of the intracellular dissolution reaction can be externalized in a continuous manner. Hence, whereas the extent of particle dissolution in solution is limited by the silica limit of solubility, the conditions of degradation within intracellular compartments can be compared to that of an open reactor, although previous reports evidenced that the exocytosis extent was dependent on the amount of silica in the medium. ${ }^{59}$ This can explain the difference evidenced for PNSPs in DMEM and within NHDFs. In the culture medium, a decrease in initial particle size is observed after 1 week and smaller particles are clearly visible at their vicinity (Figure 9). This suggests that the outer PEI/silica layers first dissolve but, because PEI is known to promote silica precipitation, ${ }^{60}$ the released soluble silica species and the polyelectrolyte chains can react together to form these new particles, as previously suggested. ${ }^{35}$ In a second stage, the largest particles turn porous, indicating that further dissolution occurs and feeds the growth of the external smaller particles. Noticeably, within the cells, these additional particles are hardly distinguished although the transformation from plain to porous large particles is also observed. This result nicely correlates with our observation that, whereas BSNPs and 
HSNPS are almost exclusively released in soluble forms during the second week, almost $30 \%$ of PNSPs are externalized in a colloidal form that may correspond to the newly-formed silica nanoparticles that are small enough to be expelled.

\section{CONCLUSION}

By following the evolution of silica nanoparticles with different internal nanostructures in solution and within normal human dermal fibroblasts, we show that their degradation pathways are very similar in all conditions although the precise composition of the medium can impact on the kinetics of the degradation. This supports previous assumptions that within such cells silica nanoparticles undergo a hydrolytic degradation process, related to silica chemistry, and not a biodegradation route, that would involve a specific biological activity. From a more fundamental point of view, these data contribute to the on-going debate on the role of silicon and its associated biochemical pathways in mammalian cells, that have a great importance to understand and predict the in vivo fate of silica biomaterials. From a practical perspective, this implies that the intracellular fate of silica-based nanomaterials can be controlled and predicted on the basis of physico-chemical considerations unless they incorporate bio-responsive functions.

\section{ASSOCIATED CONTENT}


Supporting Information. Particle size distribution of particles in water (Figure S1), DLS and zeta-metry characterization of particles during surface modification (Table S1), $\mathrm{N}_{2}$-sorption data for HSMPs (Figure S2), evolution of zeta potential with $\mathrm{pH}$ (Figure S3), fluorescence spectra of nanoparticles (Figure S4), additional TEM data for nanoparticles in culture medium (Figures S5, S6), addition fluorescence (Figures S7,S9) and TEM images (Figure S8) of NHDF after contact with nanoparticles. This material is available free of charge via the Internet at http://pubs.acs.org.

\section{AUTHOR INFORMATION}

Corresponding Author. *Email: thibaud.coradin@upme.fr

\section{ACKNOWLEDGMENTS}

Y.S. PhD grant was funded by the China Scholarship Council. The authors thank C. Aimé (LCMCP) for fruitful discussions and C. Illoul, and G. Mosser (LCMCP) for technical assistance. 


\section{REFERENCES}

(1) Slowing, I. I.; Vivero-Escoto, J. L.; Wu, C. W.; Lin, V. S. Y. Mesoporous silica nanoparticles as controlled release drug delivery and gene transfection carriers. Adv. Drug Deliv.Rev. 2008, 60, 1278-1288.

(2) Homan K. A.; Souza, M.; Truby, R.; Luke, G. P.; Green, C.; Vreeland, E.; Emelianov, S. Silver nanoplate contrast agents for in vivo molecular photoacoustic imaging. ACS Nano 2012, 6, 641-650.

(3) Yang, K.; Feng, L. Z.; Hong, H.; Cai, W. B.; Liu, Z. Preparation and functionalization of graphene nanocomposites for biomedical applications. Nature Protoc. 2013, 8, 2392-2403.

(4) Li, Z.; Wang, C.; Cheng, L.; Gong, H.; Yin, S.; Gong, Q.; Li Y.; Liu, Z. PEG-functionalized iron oxide nanoclusters loaded with chlorin e6 for targeted, NIR light induced, photodynamic therapy. Biomaterials 2013, 34, 9160-9170. 
(5) Yao, H. J.; Zhang, Y. G.; Sun L.; Liu, Y. The effect of hyaluronic acid functionalized carbon nanotubes loaded with salinomycin on gastric cancer stem cells. Biomaterials 2014, $35,9208-9223$.

(6) Jeong, Y. S.; Oh, W. K.; Kim S.; Jang, J. Cellular uptake, cytotoxicity, and ROS generation with silica/conducting polymer core/shell nanospheres. Biomaterials 2011, 32, 7217-7225.

(7) Elgrabli, D.; Dachraoui, W.; de Marmier, H.; Menard-Moyon, C.; Begin, D.; Begin-Colin, S.; Bianco, A.; Alloyeau, D.; Gazeau, F. Intracellular degradation of functionalized carbon nanotube/iron oxide hybrids is modulated by iron via Nrf2 pathway. Sci.Ren. 2017, 7, 40075.

(8) Coradeghini, R.; Gioria, S.; Garcia, C. P.; Nativo, P.; Franchini, F.; Gilliland, D.; Ponti J.; Rossi, F. Size-dependent toxicity and cell interaction mechanisms of gold nanoparticles on mouse fibroblasts. Toxicol.Lett. 2013, 217, 205-216.

(9) Hyde, E. D. E. R.; Seyfaee, A.; Neville, F.; Moreno-Atanasio, R. Colloidal Silica Particle Synthesis and Future Industrial Manufacturing Pathways: A Review. Ind. Eng. Chem. Res. 2016, 55, 8891-8913.

(10)Li, Z. X.; Barnes, J. C.; Bosoy, A.; Stoddart, J. F.; Zink, J. I. Mesoporous silica nanoparticles in biomedical applications. Chem.Soc.Rev. 2012, 41, 2590-2605.

(11)Chung, T. H.; Wu, S. H.; Yao, M.; Lu, C. W.; Lin, Y. S.; Hung, Y.; Mou, C. Y.; Chen Y. C.; Huang, D. M. The effect of surface charge on the uptake and biological 
function of mesoporous silica nanoparticles in 3T3-L1 cells and human mesenchymal stem cells. Biomaterials 2007, 28, 2959-2966.

(12) Chen, Y.; Chen, H. R.; Guo, L. M.; He, Q. J.; Chen, F.; Zhou, J.; Feng, J. W.; Shi, J. L. Hollow/rattle-type mesoporous nanostructures by a structural difference-based selective etching strategy. ACS Nano 2010, 4, 529-539.

(13) Wang Y.; Gu, H. C. Core-shell-type magnetic mesoporous silica nanocomposites for bioimaging and therapeutic agent delivery. Adv. Mater. 2015, 27, 576-585.

(14)Sun, Q.; You, Q.; Pang, X. J.; Tan, X. X.; Wang, J. P.; Liu, L.; Guo, F.; Tan, F. P.; Li, N. A photoresponsive and rod-shape nanocarrier: Single wavelength of light triggered photothermal and photodynamic therapy based on AuNRs-capped \& Ce6-doped mesoporous silica nanorods. Biomaterials 2017, 122, 188-200.

(15)Liu, T. L.; Li, L. L.; Teng, X.; Huang, X. L.; Liu, H. Y.; Chen, D.; Ren, J.; He, J. Q.; Tang, F. Q. Single and repeated dose toxicity of mesoporous hollow silica nanoparticles in intravenously exposed mice. Biomaterials 2011, 32, 1657-1668.

(16)Suma, T.; Miyata, K.; Anraku, Y.; Watanabe, S.; Christie, R. J.; Takemoto, H.; Shioyama, M.; Gouda, N.; Ishii, T.; Nishiyama, N.; Kataoka, K. Smart multilayered assembly for biocompatible siRNA delivery featuring dissolvable silica, endosome-disrupting polycation, and detachable PEG. ACS Nano 2012, 6, 6693-6705.

(17)Huang, X. L.; Teng, X.; Chen, D.; Tang, F. Q.; He, J. Q. The effect of the shape of mesoporous silica nanoparticles on cellular uptake and cell function. Biomaterials 2010, $31,438-448$. 
(18)Rosenholm, J. M.; Peuhu, E.; Eriksson, J. E.; Sahlgren, C.; Lindén, M. Targeted intracellular delivery of hydrophobic agents using mesoporous hybrid silica nanoparticles as carrier systems. Nano Lett. 2009, 9, 3308-3311.

(19)Kim, M. H.; Na, H. K.; Kim, Y. K.; Ryoo, S. R.; Cho, H. S.; Lee, K. E.; Jeon, H.; Ryoo, R.; Min, D. H. Facile synthesis of monodispersed mesoporous silica nanoparticles with ultralarge pores and their application in gene delivery. ACS Nano 2011, 5, 3568-3576.

(20) Ratcliffe, S.; Jugdaohsingh, R.; Vivancos, J.; Marron, A.; Deshmukh, R.; Ma, J. F.; Mitani-Ueno, N.; Robertson, J.; Wills, J.; Boekschoten, M. V.; Müller, M.; Mawhinney, R. C.; Kinrade, S. D.; Isenring, P.; Bélanger, R. R.; Powell, J. J. Identification of a mammalian silicon transporter. Am.J.Physiol.Cell Phvsiol. 2017, 312, C550-C561.

(21) Croissant, J. G.; Fatieiev, Y.; Kashab, N. M. Functional Nanoparticles: Degradability and Clearance of Silicon, Organosilica, Silsesquioxane, Silica Mixed Oxide, and Mesoporous Silica Nanoparticles. Adv.Mater. 2017, 29, 1604634

(22)Braun, K.; Pochert, A.; Beck, M.; Fiedler, R.; Gruber, J.; Lindén, M. Dissolution kinetics of mesoporous silica nanoparticles in different simulated body fluids. J. Sol-Gel Sci. Technol. 2016, 79, 319-327.

(23) Yang, Y.; Coradin, T. A green route to silica nanoparticles with tuneable size and structure. Green Chem. 2008, 10, 183-190. 
(24)Quignard, S.; Masse, S.; Laurent, G.; Coradin, T. Introduction of disulfide bridges within silica nanoparticles to control their intra-cellular degradation. Chem. Commun. 2013, $49,3410-3412$.

(25)Corsi, F.; De Palma, C.; Colombo, M.; Allevi, R.; Nebuloni, M.; Ronchi, S.; Rizzi, G.; Tosoni, A.; Trabucchi, E.; Clementi, E.; Prosperi, D. Towards Ideal Magnetofluorescent Nanoparticles for Bimodal Detection of Breast-Cancer Cells. Small 2009, 5, 2555-2564.

(26) Dove, P. M.; Han, N.; Wallace, A. F.; De Yoreo, J. J. Kinetics of amorphous silica dissolution and the paradox of silica polymorphs. Proc. Natl. Acad. Sci. USA 2008, 105, 9903-9908.

(27) Iler, R. K. The Chemistry of Silica: Solubility, Polymerization, Colloid and Surfaces Properties, and Biochemistry; Wiley: New York,1979.

(28) Ehrlich, H.; Demadis, K. D.; Pokrovsky, O. S.; Koutsoukos, P. G. Modern Views on Desilicification: Biosilica and Abiotic Silica Dissolution in Natural and Artificial Environments. Chem. Rev. 2000, 110, 4656-4689.

(29)Viitala, R.; Jokinen, M.; Tuusa, S.; Rosenholm, J. B.; Jalonen, H. Adjustably bioresorbable sol-gel derived $\mathrm{SiO}_{2}$ matrices for release of large biologically active molecules. L.Sol-Gel Sci.Technol. 2005, 36, 147-156.

(30) Finnie, K. S.; Waller, D. J.; Perret, F. L.; Krause-Heuer, A. M.; Lin, H. Q.; Hanna, J. V.; Barbé, C. J. Biodegradability of sol-gel silica microparticles for drug delivery. $J$. Sol-Gel Sci. Technol. 2009, 49, 12-18. 
(31) Yamada, H.; Urata, C.; Aoyama, Y.; Osada, S.; Yamauchi, Y.; Kuroda, K. Preparation of Colloidal Mesoporous Silica Nanoparticles with Different Diameters and Their Unique Degradation Behavior in Static Aqueous Systems. Chem.Mater. 2012, 24, 1462-1471.

(32) Bass, J. D.; Grosso, D.; Boissière, C.; Belamie, E.; Coradin, T.; Sanchez, C. Stability of Mesoporous Oxide and Mixed Metal Oxide Materials in Biologically Relevant Conditions. Chem. Mater. 2007, 19, 4349-4356.

(33) Galarneau, A.; Nader, M.; Guenneau, F.; Di Renzo, F.; Gedeon, A. Understanding the Stability in Water of Mesoporous SBA-15 and MCM-41. J. Phys. Chem. C 2007, 111, 8268-8277.

(34)He, Q. J.; Shi, J. L.; Zhu, M.; Chen, Y.; Chen, F. The three-stage in vitro degradation behavior of mesoporous silica in simulated body fluid. Microp. Mesop. Mater. 2010, 131, 314-320.

(35)Chen, K.; Zhang, J.; Gu, H. Dissolution from inside: a unique degradation behaviour of core-shell magnetic mesoporous silica nanoparticles and the effect of polyethyleneimine coating. L.Mater. Chem. 2012, 22, 22005-22012.

(36) Boissière, M.; Meadows, P. J.; Brayner, R.; Hélary, C.; Livage, J.; Coradin, T. Turning biopolymer particles into hybrid capsules: the example of silica/alginate nanocomposites. L.Mater. Chem. 2006, 16, 1178-1182.

(37) Allouche, J.; Boissière, M.; Hélary, C.; Livage, J.; Coradin, T. Biomimetic core-shell gelatine/silica nanoparticles: a new example of biopolymer-based nanocomposites. J. Mater. Chem. 2006, 16, 3121-3126. 
(38) Slowing I. I.; Vivero-Escoto, J. L.; Zhao, Y.; Kandel, K.; Peeraphatdit, C.; Trewyn, B. G.; Lin, V.S. Exocytosis of mesoporous silica nanoparticles from mammalian cells: from asymmetric cell-to-cell transfer to protein harvesting. Small 2011, 7, 1526-1532.

(39) Quignard, S.; Mosser, G.; Boissière, M.; Coradin, T. Long-term fate of silica nanoparticles interacting with human dermal fibroblasts. Biomaterials 2012, 33, 4431-4442.

(40) Zhai, W.; He, C.; Wu, L.; Zhou, Y.; Chen, H.; Chang, J.; Zhang, H. Degradation of hollow mesoporous silica nanoparticles in human umbilical vein endothelial cells. $J$. Biomed. Mater.Res.B 2012, 100B, 1397-1403.

(41) Chen, G.; Teng, Z.; Su, X.; Liu, Y.; Lu, G. Unique Biological Degradation Behavior of Stöber Mesoporous Silica Nanoparticles from Their Interiors to Their Exteriors. J. Biomed. Nanotechnol. 2015, 11, 722-729.

(42) Kempen, P. J.; Greasley, S.; Parker, K. A.; Campbell, J. L.; Chang, H.-Y.; Jones, J. R.; Sinclair, R.; Gambhir, S. S.; Jokerst, J. V. Theranostic Mesoporous Silica Nanoparticles Biodegrade after Pro-Survival Drug Delivery and Ultrasound/Magnetic Resonance Imaging of Stem Cells. Theranostics 2015, 5, 631-642.

(43)Bergman, L.; Kankaanpää, P.; Tiitta, S.; Duchanoy, A.; Ji, L.; Heino, J.; Lindén, M. Intracellular Degradation of Multilabeled Poly(Ethylene imine)-Mesoporous Silica-Silica Nanoparticles: Implications for Drug Release. Mol.Pharm. 2013, 10, 1795-1803 
(44) Couleaud, P.; Morosini, V.; Frochot, C.; Richeter, S.; Raehm, L.; Durand, J. O. Silica-based nanoparticles for photodynamic therapy applications. Nanoscale 2010, 2, 1083-1095.

(45)Montalti, M.; Prodi, L.; Rampazzo, E.; Zaccheroni, N. Dye-doped silica nanoparticles as luminescent organized systems for nanomedicine. Chem.Soc.Rev. 2014, 43, 4243-4268.

(46) Li, Z.; Yuan, D.; Jin, G.; Tan, B.; He, C. Facile Layer-by-Layer Self-Assembly toward Enantiomeric Poly(lactide) Stereocomplex Coated Magnetite Nanocarrier for Highly Tunable Drug Deliveries. ACS Anpl. Mater.Interfaces 2016, 8, 1842-1853.(47) Mebert, A. M.; Aimé, C.; Alvarez, G. S.; Shi, Y.; Flor, S. A.; Lucangioli, S. E.; Desimone, M. F.; Coradin, T. Silica core-shell particles for the dual delivery of gentamicin and rifamycin antibiotics. L.Mater. Chem. B 2016, 4, 3135-3144.

(48) Andersson, J.; Rosenholm, J.; Areva, S.; Lindén, M. Influences of Material Characteristics on Ibuprofen Drug Loading and Release Profiles from Ordered Micro- and Mesoporous Silica Matrices. Chem.Mater. 2004, 16, 4160-4167

(49)Bouledjouidja, A.; Masmoudi, Y.; Van Speybroeck, M.; Schueller, L. Badens, E. Impregnation of Fenofibrate on mesoporous silica using supercritical carbon dioxide. Int. J. Pharm. 2016, 499, 1-9.

(50) Zhou, Z.; Zhang, C.; Qian, Q.; Ma, J.; Huang, P.; Zhang, X.; Pan, L.; Gao, G.; Fu, H.; Fu, S.; Song, H.; Zhi, X.; Ni, J.; Cui, D. Folic acid-conjugated silica capped gold nanoclusters for targeted fluorescence/X-ray computed tomography imaging. $J$. Nanobiotechnol. 2013, 11, 17. 
(51) Nakamura, T.; Sugihara, F.; Matsushita, H.; Yoshioka, Y.; Mizukami, S.; Kikuchi, K. Mesoporous silica nanoparticles for ${ }^{19} \mathrm{~F}$ magnetic resonance imaging, fluorescence imaging, and drug delivery. Chem. Sci. 2015, 6, 1986-1990.

(52) van Blaaderen, A.; Vrij, A. Synthesis and characterization of colloidal dispersions of fluorescent, monodisperse silica spheres. Langmuir 1992, 8, 2921-2931.

(53) Chen, F.; Hong, H.; Shi, S. X.; Goel, S.; Valdovinos, H. F.; Hernandez, R.; Theuer, C. P.; Barnhart, T. E.; Cai, W. B. Engineering of Hollow Mesoporous Silica Nanoparticles for Remarkably Enhanced Tumor Active Targeting Efficacy. Sci.Rep. 2014, 4, 5080.

(54) Wang, X.; Masse, S.; Laurent, G.; Hélary, C.; Coradin, T. Impact of Polyethylenimine Conjugation Mode on the Cell Transfection Efficiency of Silica Nanovectors. Langmuir 2015, 31, 11078-11085.

(55) Drescher, D.; Orts-Gil, G.; Laube, G.; Natte, K.; Veh, R. W.; Österle, W.; Kniepp, J. Toxicity of amorphous silica nanoparticles on eukaryotic cell model is determined by particle agglomeration and serum protein adsorption effects. Anal. Bioanal. Chem. 2011, 400, 1367-1373

(56) Noble, J. E.; Wang, L.; Cole, K. D.; Gaigalas, A. K. The effect of overhanging nucleotides on fluorescence properties of hybridising oligonucleotides labelled with Alexa-488 and FAM fluorophores. Biophvs. Chem. 2005, 113, 255-263. 
(57) Soulé, S.; Bulteau, A. L.; Faucher, S.; Haye, B.; Aimé, C.; Allouche, J.; Dupin, J. C.;

Lespes, G.; Coradin, T.; Martinez, H. Design and Cellular Fate of Bioinspired Au-Ag Nanoshells@hybrid Silica Nanoparticles._Lanomuir 2016, 32, 10073-10082.

(58) Tappel, A. L.; Shibko, S.; Stein, M.; Susz, J. P. Studies on the Composition of Lysosomes. J.Food.Sci. 1965, 30, 498-503.

(59) Chu, Z.; Huang, Y.; Tao, Q.; Li, Q. Cellular uptake, evolution, and excretion of silica nanoparticles in human cells. Nanoscale 2011, 3, 3291-3299.

(60) Demadis, K. D.; Pachis, K.; Ketsetzi, A.; Stathoulopoulou, A. Bioinspired control of colloidal silica in vitro by dual polymeric assemblies of zwitterionic phosphomethylated chitosan and polycations or polyanions. Adv. Colloid Interface Sci. 2009, 151, 33-48. 


\section{TOC graphics}

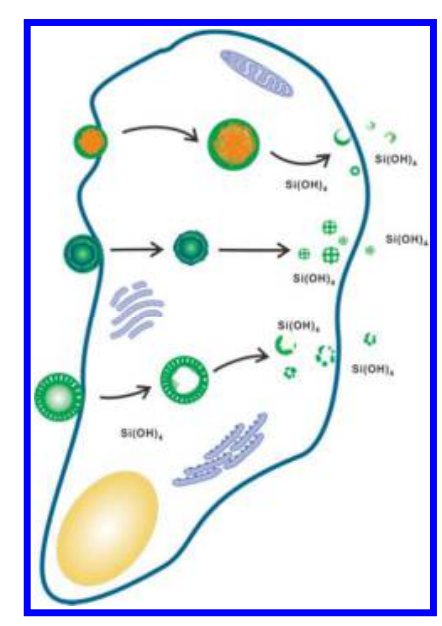

